\title{
P-0972 - Is metformin in first trimester of pregnancy safe for the mother and fetus in gestational diabetes mellitus?
}

R. Dasgupta ${ }^{1}, M$. Vanlalhruaii ${ }^{1}$, R. Ramachandran ${ }^{1}, J$. Mathews ${ }^{2}, A$. Regi ${ }^{2}$,V. Jeyaseelan ${ }^{3}$, A. Hesarghatta $S^{1}, N$. Thomas ${ }^{1}$ ${ }^{1}$ Department of Endocrinology, ${ }^{2}$ Obstetrics and Gynecology, ${ }^{3}$ Biostatistics, Christian Medical College Vellore, India.

AIMS AND OBJECTIVES :

- In women with Gestational Diabetes Mellitus(GDM):

$>$ Assess the maternal and fetal outcomes with use of metformin in the first trimester of pregnancy

$>$ Compare these outcomes with initiation of metformin after the first trimester of pregnancy or initiation of insulin alone during any trimester

MATERIALS AND METHODS:

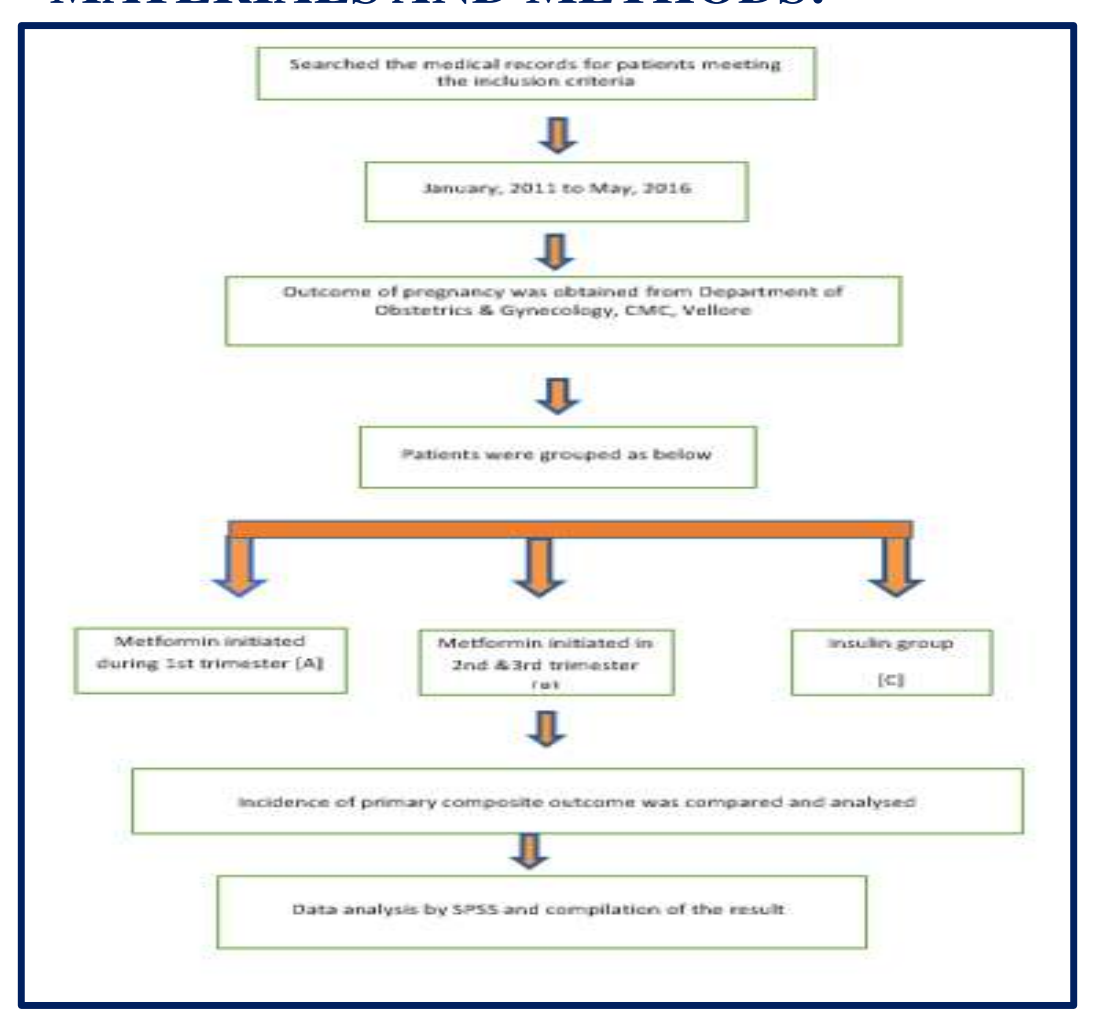

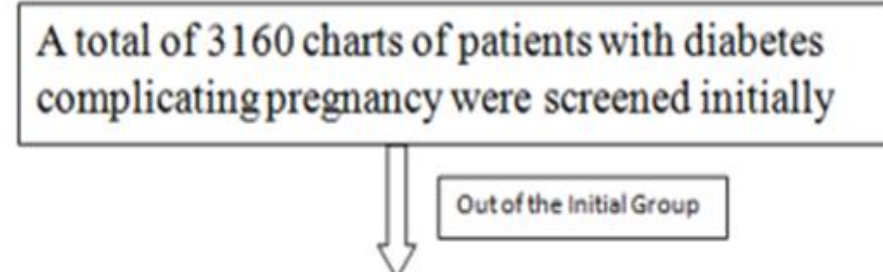

1368 patients had Pre-gestational Diabetes

788 did not fit either one or more of the inclusion criteria

464 incomplete set of data on retrospective chart review

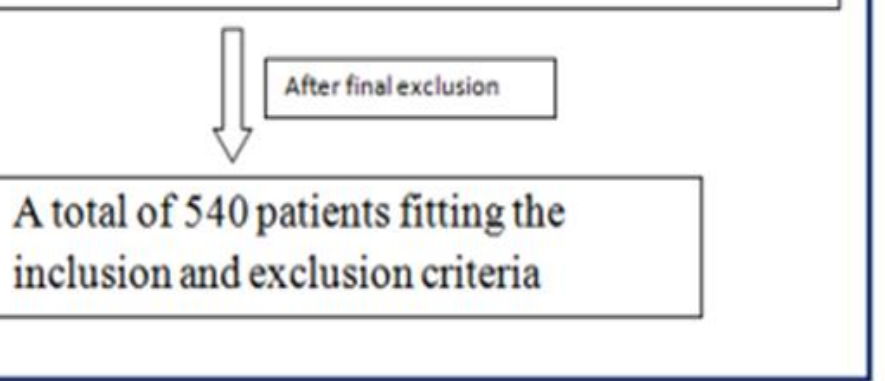

Primary outcome measures:

- Neonatal hypoglycemia

-Respiratory distress

-Need for phototherapy

-Birth trauma

-5-minute APGAR score $<7$

-Prematurity

Inclusion criteria -

Women with GDM or overt DM

Exclusion criteria

Pre-gestational diabetes or Hypertension

GDM women only on Medical Nutrition

Therapy
RESULTS AND ANALYSIS :

\section{BASELINE CHARACTERISTICS}

Age of subject (years)

Gestational age at diagnosis (weeks + days)

0 hour plasma glucose(OGTT, in $\mathrm{mg} \%$ )

2 hours plasma glucose(OGTT, in $\mathrm{mg} \%$ )

History of GDM (\%)

Family history of diabetes mellitus (\%)

Duration of hospital stay during delivery (days)

Pregnancy loss (\%)

Pre eclampsia (\%)

Birth length $(\mathrm{cm})$

Gestational age at delivery (weeks + days)

\begin{tabular}{|l|l|l|l|}
\hline $\begin{array}{l}\text { Composite outcome } \\
\text { (Group A \& B) }\end{array}$ & Value & Lower CI (95\%) & Upper CI (95\%) \\
\hline Primary outcome (OR) & 1.738 & 0.657 & 4.597 \\
\hline Primary outcome yes & 1.633 & 0.685 & 3.891 \\
\hline Primary outcome no & 0.939 & 0.843 & 1.046 \\
\hline Secondary outcome (OR) & 1.617 & 0.618 & 4.115 \\
\hline Secondary outcome- yes & 1.702 & 0.689 & 3.651 \\
\hline Secondary outcome- no & 0.840 & 0.850 & 1.00 \\
\hline
\end{tabular}

\begin{tabular}{|l|l|l|l|}
\hline Composite outcome & Value & Lower Cl (95\%) & Upper Cl (95\%)
\end{tabular}

\section{(Group A \& C)}

Primary outcome (OR)

Primary outcome yes

Primary outcome no

Secondary outcome (OR)

Secondary outcome- yes

Secondary outcome- no

Subjects $(\mathrm{N}=540)$

$29.06 \pm 4.64$

$21.8 \pm 8.6$

$122.36 \pm 43.05$

$202.44 \pm 108.98$

$121 \pm 22.4$

$311 \pm 61.29$

$5.54 \pm 2.76$

$14 \pm 2.59$

$12 \pm 2.22$

$47.90 \pm 2.61$

$38.49 \pm 5.60$
CONCLUSIONS:

$>$ Our findings strongly suggest that metformin use in the first ${ }^{t}$ trimester has no significant maternal or fetal adverse outcomes

$>$ Prematurity and fetal loss are two outcomes that warrant further critical evaluation in future studies in larger cohorts of GDM.

$>$ Metformin can prove to be a safe, effective and cheaper modality of treatment in the first trimester of gestation in GDM mothers .
Individual outcome comparison : Group A\&B and Group A\&C

\begin{tabular}{|c|c|c|c|c|c|}
\hline \multirow{2}{*}{ Variables } & \multicolumn{2}{|c|}{ Total subjects $=389$} & \multirow[t]{2}{*}{ P-value } & \multirow{2}{*}{$\begin{array}{l}\text { Group C } \\
(\mathrm{N}-151)\end{array}$} & \multirow{2}{*}{ P-value } \\
\hline & $\begin{array}{l}\text { Group A } \\
(\mathrm{N}=186)(\%)\end{array}$ & $\begin{array}{l}\text { GroupB } \\
(N=203)(\%)\end{array}$ & & & \\
\hline Neonatal hypoglycemia & $3(1.61 \%)$ & 0 & 0.203 & $2(1.32 \%)$ & 0.204 \\
\hline Respiratory distress & $1(0.53 \%)$ & 0 & 0.377 & 0 & 0.368 \\
\hline Need for phototherapy & $1(0.53 \%)$ & $2(0.98 \%)$ & 0.876 & $1(0.66 \%)$ & 0.886 \\
\hline Birth trauma & $1(0.53 \%)$ & 0 & 0.534 & $1(0.66 \%)$ & 0.530 \\
\hline 5 minutes APGAR $<7$ & 0 & $2(0.98 \%)$ & 0.193 & 0 & 0.197 \\
\hline Premature birth & $18(9.67 \%)$ & $14(6.89 \%)$ & 0.540 & $14(9.27 \%)$ & 0.537 \\
\hline Gestational HTN & $158(84.94 \%)$ & $181(89.16 \%)$ & 0.101 & $140(92 \%)$ & 0.104 \\
\hline PPGT $=\#$ & $10(12.98 \%)$ & $14(18.8 \%)$ & 0.227 & $14(9.3 \%)$ & 0.232 \\
\hline $\begin{array}{l}\text { Optimal Maternal } \\
\text { alxcemosic control ** }\end{array}$ & $149(92.5 \%)$ & $144(90 \%)$ & 0.661 & $110(92 \%)$ & 0.659 \\
\hline Birth weight $<4 \mathrm{kgs}$ & $183(90.14 \%)$ & $198(97.53 \%)$ & 0.462 & $146(96 \%)$ & 0.465 \\
\hline Birth length $<50 \mathrm{C}-\mathrm{ms} \wedge$ & $106(87.6 \%)$ & $129(92.8 \%)$ & 0.273 & $45(36.5 \%)$ & 0.277 \\
\hline
\end{tabular}

\begin{tabular}{|c|c|c|c|c|c|}
\hline \multicolumn{3}{|c|}{$\begin{array}{l}\text { PREGNANCY LOSS } \\
\text { COMPARISON }\end{array}$} & $\begin{array}{l}\text { Group } \\
A(N=11)\end{array}$ & $\begin{array}{l}\text { Group } \\
B(N=1)\end{array}$ & $\begin{array}{l}\text { Group } \\
\mathrm{C}(\mathrm{N}=2)\end{array}$ \\
\hline \multicolumn{3}{|c|}{ Spontaneous abortion } & $1(9.1 \%)$ & 0 & 0 \\
\hline \multirow[t]{4}{*}{ MTP } & IUD & Still birth & 6 (54.5\%) & $1(100 \%)$ & 0 \\
\hline & \multirow[t]{3}{*}{ Anomaly } & CVS & 1 (9.1\%) & 0 & $1(50 \%)$ \\
\hline & & NS & $2(18.2 \%)$ & 0 & $1(50 \%)$ \\
\hline & & Rhizomelia & $1(9.1 \%)$ & 0 & 0 \\
\hline Total & & & 11 & 1 & 2 \\
\hline
\end{tabular}

Key Findings:

$>$ Premature birth numerically higher $(9.9 \%)$ in Group A compared to Group B (6.9\%) patients and Group C (9.3\%) (P-Value 0.540, 0.537)- similar to MiG Trial (12.1\%)

$>$ No other primary or secondary composite outcome comparison showed statistically significant difference 